\title{
ESTUDO DE CASO: EMPREGO DA MATRIZ DE LEOPOLD PARA A AVALIAÇÃO DE IMPACTOS ASSOCIADOS À PRODUÇÃO DE TABACO EM UMA PROPRIEDADE NO VALE DO RIO PARDO
}

\author{
Douglas Falk ${ }^{1}$, Aline Rubert ${ }^{1}$, Luana Schlesener Voese ${ }^{1}$, Maiara Priscilla de Souza ${ }^{2}$, Rosana de Cassia de Souza Schneider ${ }^{1-2 *}$ \\ 1 Curso de Graduação em Química - Linha de formação Química Industrial, Universidade de Santa Cruz do Sul (UNISC), CEP \\ 96815-900, Santa Cruz do Sul - RS, Brasil. \\ 2Programa de Pós-graduação em Tecnologia Ambiental, Universidade de Santa Cruz do Sul (UNISC), CEP 96815-900, Santa Cruz \\ do Sul - RS, Brasil.
}

*E-mail: rosana@unisc.br

\section{RESUMO}

A indústria de tabaco vem ganhando destaque com o passar dos anos, no entanto, suas consequências para a saúde continuam sendo uma preocupação constante. Considerando o destaque dessas indústrias, os sistemas de gestão são imprescindíveis para minimizar impactos neste e em outros setores, considerando os aspectos relacionados a sustentabilidade de um processo. Devido às consequências que podem trazer ao meio ambiente, este trabalho visa efetuar um estudo de caso de uma propriedade produtora de tabaco a fim de determinar impactos ambientais usando uma matriz de interação. A Matriz de Leopold foi o modelo utilizado para avaliar os efeitos bióticos e antrópicos em comparação aos aspectos ambientas. Os resultados encontrados demostraram os pontos mais críticos, importantes, frequentes e significativos de cada etapa do processo de produção agrícola de tabaco e que são de extrema importância ambiental, pois causam impactos negativos no ar, solo e água. Com a avaliação realizada identificou-se que os impactos positivos $(43,8 \%)$ estão relacionados as atividades antrópicas e os negativos $(56,3 \%)$ estão relacionados principalmente ao meio físico.

Palavras-chave: Tabaco. Impactos Ambientais. Matriz de Leopold.

\section{Introdução}

Atualmente, a indústria do tabaco se organiza em nível mundial sob a forma de oligopólio, onde poucas empresas detêm o controle de grande parcela do mercado. As atividades de produção e comercialização de tabaco e seus derivados se estendem por mais de cinquenta países. Os maiores produtores são China (2,83 milhões de toneladas), Brasil (0,85 milhões de toneladas), e Estados Unidos (0,52 milhões de toneladas) [1].

No Brasil, a Região Sul se destaca na produção de tabaco e de cigarros, sendo $25 \%$ da produção de tabaco do sul do Brasil proveniente do Vale do Rio Pardo, e Santa Cruz do Sul um dos principais municípios do setor fumageiro. Esse município exporta cerca de $85 \%$ de sua produção [2]. Nessa região, aproximadamente $50 \%$ da sua população vive no meio rural, dedicando-se principalmente à produção do tabaco [3].

As indústrias de tabaco são responsáveis por grande parcela de empregos na região, ainda assim, a cadeia produtiva desses estabelecimentos deve ser sempre estudada a fim de minimizar danos aos diversos meios, como, por exemplo, a contaminação dos alimentos, do solo, da fauna, dos rios, desmatamento e perda de biodiversidade, uma vez que os pacotes tecnológicos de agrotóxicos podem mudar, dependendo das pragas e dos produtos disponíveis no comércio [1]. Além disso, as famílias de agricultores que cultivam fumo são sistematicamente afetadas de diversas formas, tais como: uso de agrotóxicos; pelo contato direto com a planta úmida, que libera nicotina, sendo esta absorvida pela epiderme; cheiro e poeira das folhas durante a secagem nas estufas, dentre outros. O uso de agrotóxicos é o mais complexo e abrangente, pois além de atingir o solo e água, pode afetar a camada de ozônio, e alguns dos agrotóxicos utilizados são encontrados inclusive no produto final da atividade agrícola, ou seja, na folha [4-5].

Outra problemática ambiental é o uso do sistema Floating para produção das mudas. Este processo consiste em flutuar bandejas de poliestireno, as quais são enchidas com substrato, numa lâmina d'água em um túnel coberto por plástico. Nessa lâmina, são diluídos os agrotóxicos e os fertilizantes, formando um concentrado químico [9-10]. Essas soluções apresentam toxicidade e, na maioria das vezes, seja na forma pura ou misturada com calcário, são descartadas diretamente no 
solo, ou permanecem sistemas até evaporar completamente ou serem carreados pelas águas das chuvas [1].

Uma das mais populares ferramentas de avaliação de impactos ambientais é a Matriz de Leopold. Esta matriz passou por diversas adaptações e tem sido utilizada de variadas formas ao longo do tempo, dependendo do processo a ser avaliado. A Matriz de Leopold possibilita a avaliação das interações entre as ações realizadas sobre determinado aspecto ambiental, como por exemplo biológico, físico, químico ou antrópico [10].

Diante da relevância do tema para a região, e de sua contribuição para estudos futuros em locais que trabalham com a cultura do tabaco, este estudo visa analisar os impactos ambientais relacionados à produção de tabaco em uma propriedade do Vale do Rio Pardo, com uso da matriz de Leopold modificada. O objetivo foi correlacionar os aspectos ambientais com efeitos bióticos e antrópicos, avaliando as interações entre ações da cultura do tabaco e fatores ambientais, classificando impactos ambientais potenciais, sendo analisadas questões referentes à significância, frequência, importância e criticidade de cada etapa do processo.

\section{Metodologia}

Para o estudo de caso foi realizada a visitação em uma propriedade agrícola que tem tradição de 30 anos na produção de folhas de tabaco. As ações realizadas na lavoura estudada são similares àquelas realizadas em outras propriedades da região. A matriz de Leopold modificada [11] foi usada para identificar as principais atividades e seus impactos ambientais durante a produção do tabaco no Vale do Rio Pardo. Os fatores ambientais analisados definidos para monitoramento são: Físicos; 1. Recurso atmosférico (partículas sólidas e gases); 2. Recurso hídrico (contaminação); 3. Recurso edáfico (contaminação dos solos); 4. Antrópicos (economia local, infraestrutura necessária, qualidade de vida e saúde, qualidade do produto final). Foram analisadas as seguintes etapas do processo: produção e preparo das mudas, preparação do solo, plantio, capação, colheita, cura das folhas, classificação, manocas e enfartamento e transporte até as indústrias.

Cada fator, ação identificada no processo e impactos ambientais foram determinados com base nas seguintes classificações: valor (positivo ou negativo); ordem (direta ou indireta); espaço (local, regional ou estratégico); tempo (curto, médio ou longo); dinâmica (temporária, cíclica ou permanente). Para classificar a etapa do processo de acordo com os critérios enumerados anteriormente, foram utilizados os seguintes critérios: pesquisas na literatura e entrevistas com produtores, buscando coletar informações e aliá-las a conhecimentos prévios, como, por exemplo, o impacto do uso de herbicidas e outros compostos orgânicos potencialmente tóxicos. Como intervalo de avaliação, utilizou-se desde a etapa da produção de mudas até a entrega para a indústria. A Tabela 1 descreve os critérios utilizados para classificar as interações entre os fatores e as etapas de produção.

Tabela 1. Descrição dos critérios utilizados para identificar as principais atividades e seus impactos ambientais durante a produção do tabaco na propriedade em estudo, elaborada pelos autores.

\begin{tabular}{|c|c|}
\hline Classificação & Descrição \\
\hline N-Negativo & $\begin{array}{l}\text { Polui/contamina o fator ambiental em questão ou gera } \\
\text { algum tipo de risco para quem participa de tal etapa. }\end{array}$ \\
\hline P-Positivo & $\begin{array}{l}\text { Agrega valor ao produto final. Não gera risco significativo } \\
\text { no processo. }\end{array}$ \\
\hline D-Direto & Afeta diretamente o fator ambiental \\
\hline I-Indireto & Afeta de modo indireto o fator ambiental \\
\hline L-Local & Impacta apenas no local da propriedade de produção \\
\hline R-Regional & Impacta sobre toda a região de produção \\
\hline C-Curto & $\begin{array}{l}\text { Ocorre em um curto espaço de tempo ou gera um efeito de } \\
\text { curta duração }\end{array}$ \\
\hline M-Médio & $\begin{array}{l}\text { Ocorre em um médio espaço de tempo ou gera um efeito de } \\
\text { média duração }\end{array}$ \\
\hline X-Longo & $\begin{array}{l}\text { Ocorre em um longo espaço de tempo ou gera um efeito de } \\
\text { longa duração }\end{array}$ \\
\hline Y-Permanente & $\begin{array}{l}\text { Impacta em todo ciclo de produção ou de modo permanente } \\
\text { a quem é exposto ao risco }\end{array}$ \\
\hline T-Temporário & $\begin{array}{l}\text { Impacto somente em uma pequena parte do ciclo ou em } \\
\text { quem participa da etapa de produção }\end{array}$ \\
\hline
\end{tabular}

\section{Resultados e discussões}

A avaliação do processo de produção do tabaco foi realizada através do monitoramento de 8 etapas principais, apresentadas na Tabela 2.

A Tabela 3 mostra a matriz de interações entre os fatores ambientais e as ações no processo de produção do tabaco. Foram avaliadas 150 possíveis interações, sendo 61 delas identificadas. Nas colunas, encontram-se os fatores ambientais com potencial de serem impactados; nas linhas, as etapas do processo de produção do tabaco.

$\mathrm{Na}$ Figura 1, são apresentados os gráficos gerados a partir dos resultados encontrados na Tabela 3. Os gráficos mostram os resultados obtidos na avaliação das classificações do impacto da etapa produtiva no fator ambiental. Analisando os gráficos, podemos ver que a maioria das etapas possui impacto: negativo, direto, permanente, local e longo.

De acordo com os dados obtidos, pôde-se compreender como a cultura do tabaco influencia no meio ambiente, principalmente em relação aos impactos negativos nos recursos hídricos, no ar e no solo. Além disso, o agricultor participa de todo processo, estando exposto a riscos à saúde associados à atividade de produção do tabaco, como partículas em suspensão, altas temperaturas (estufas), compostos altamente tóxicos e acumulativos (agrotóxicos), entre outros. 
Tabela 2. Etapas e observações registradas durante as visitações a propriedade agrícola em estudo.

\begin{tabular}{|c|c|}
\hline Etapa & Observação \\
\hline 1. Produção e preparo das mudas & $\begin{array}{l}\text { Consiste na obtenção de mudas sadias e produtivas para o processo } \\
\text { de plantio. Nesta etapa, pode-se destacar a importância e a } \\
\text { influência na qualidade do produto final, ou seja, mudas que } \\
\text { apresentaram algum problema oriundo de baixo desenvolvimento } \\
\text { causam consequentemente perda da qualidade no produto final. }\end{array}$ \\
\hline 2. Preparação do Solo & $\begin{array}{l}\text { Consiste nos processos de calagem, preparo físico (lavragem, } \\
\text { gradeamento), aplicação de herbicidas e/ou fungicidas. } \\
\text { Basicamente, trata-se da adequação do solo para gerar maior } \\
\text { produtividade. Também de grande importância para qualidade, a } \\
\text { calagem pode gerar material particulado suspenso no ar quando } \\
\text { aplicado de modo equivocado. Já os impactos dos herbicidas e } \\
\text { fungicidas já são bem conhecidos, contaminando rios, solos e o } \\
\text { próprio ar, além de ser um grande risco à saúde do trabalhador [12] }\end{array}$ \\
\hline 3. Plantio & $\begin{array}{l}\text { Consiste na colocação das mudas no solo. Necessita um } \\
\text { permanente controle de pragas e adubação de cobertura, as quais } \\
\text { geram impactos sobre o solo, ar e água, além de prejudicar a saúde } \\
\text { do trabalhador, conforme discutido anteriormente. Nesta etapa há a } \\
\text { aplicação de agrotóxicos, aos quais muitos produtores utilizam sem } \\
\text { o cuidado adequado. Estudos recentes relacionaram a perda da } \\
\text { qualidade de audição ao uso de agrotóxicos pelos produtores rurais } \\
\text { [13]. Além disso, a contaminação por agrotóxicos pode se espalhar } \\
\text { por regiões circunvizinhas por meio dos corpos d'agua. Por se tratar } \\
\text { de poluentes orgânicos persistentes, podem permanecer no meio } \\
\text { ambiente por muito tempo [14], podendo chegar até aos alimentos } \\
\text { e à água de consumo. }\end{array}$ \\
\hline 4. Capação & $\begin{array}{l}\text { Trata-se da quebra do botão floral e da aplicação de antibrotante, } \\
\text { um produto de toxicidade considerável, podendo gerar } \\
\text { contaminações no solo, ar, água e no indivíduo que o aplica. }\end{array}$ \\
\hline 5. Colheita & $\begin{array}{l}\text { Retirada das folhas da planta. Possui um elevado risco ao } \\
\text { trabalhador, pois muitas folhas se localizam em partes que exigem } \\
\text { do produtor uma postura desconfortável para a realização da } \\
\text { colheita. Há de se considerar também que muitas folhas estão } \\
\text { contaminadas com agrotóxicos utilizados anteriormente. Há } \\
\text { transporte da lavoura para a propriedade com consumo de } \\
\text { combustível. }\end{array}$ \\
\hline 6. Cura das folhas & $\begin{array}{l}\text { Secagem em estufa das folhas colocadas sobre uma vara de } \\
\text { sustentação, para adquirirem as propriedades necessárias. A } \\
\text { secagem gera gases e vapores nocivos, embora possua grande } \\
\text { importância para a qualidade do produto final. }\end{array}$ \\
\hline 7. Classificação, manocas e enfartamento & $\begin{array}{l}\text { Classificação do tabaco em grupos de acordo com seu preparo, sua } \\
\text { apresentação e arrumação, sua posição nas plantas, cor das folhas e } \\
\text { sua qualidade. Possui grande importância econômica e na qualidade } \\
\text { do produto final, mas gera grande quantidade de poluição no ar, } \\
\text { devido ao forte cheiro, e material particulado. Há risco ao } \\
\text { trabalhador, uma vez que muitos realizam este processo em posturas } \\
\text { com pouca ergonomia, em assentos inapropriados para um correto } \\
\text { acomodamento da coluna vertebral. }\end{array}$ \\
\hline 8. Transporte até as indústrias & $\begin{array}{l}\text { Possui impacto na economia visto que necessita de mão de obra para } \\
\text { ser realizado e há consumo de combustíveis. }\end{array}$ \\
\hline
\end{tabular}




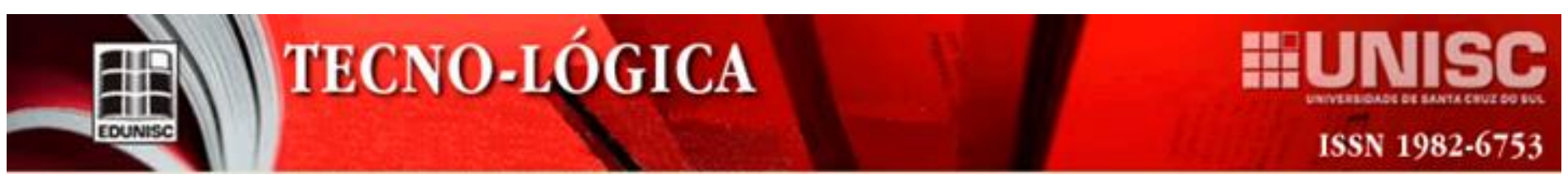

Tabela 3. Matriz de Leopold modificada para análise dos impactos ambientais.

\begin{tabular}{|c|c|c|c|c|c|c|c|c|c|}
\hline \multirow[b]{4}{*}{ Etapas } & \multicolumn{8}{|c|}{ CARACTERÍSTICAS AMBIENTAIS RELEVANTES } & \\
\hline & & & Me & 'ísico & & \multirow[b]{3}{*}{ 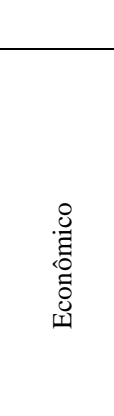 } & \multicolumn{2}{|c|}{ Meio Antrópico } & \multirow[b]{3}{*}{ 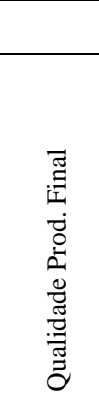 } \\
\hline & & & & $\begin{array}{l}\text { Recurso } \\
\text { Hídrico }\end{array}$ & $\begin{array}{l}\text { Recurso } \\
\text { Edáfico }\end{array}$ & & \multirow{2}{*}{\multicolumn{2}{|c|}{ 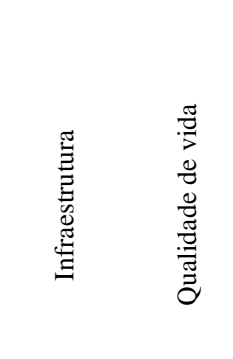 }} & \\
\hline & Atividades & 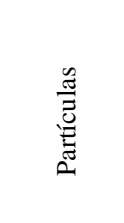 & 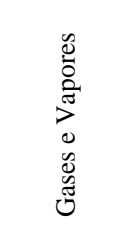 & 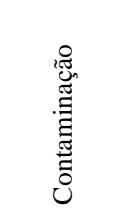 & 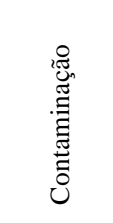 & & & & \\
\hline \multirow{4}{*}{$\begin{array}{c}\text { Produção e } \\
\text { preparo das } \\
\text { mudas }\end{array}$} & Construção dos canteiros & - & - & - & - & PIRCT & PDLMT & - & - \\
\hline & Obtenção de mudas e bandejas & - & - & - & - & PDRMT & PDLMT & - & PDLXY \\
\hline & Preparo do substrato & - & - & - & - & PDLCT & - & - & PDLMT \\
\hline & Repique & - & - & - & - & PDLCT & - & NDXY & PDLXY \\
\hline \multirow{3}{*}{$\begin{array}{l}\text { Preparação do } \\
\text { solo }\end{array}$} & Calagem & NDLCT & - & NILXT & NDLXY & PIRCT & - & - & PDLXY \\
\hline & Lavragem/gradeamento & - & - & - & - & PDRCT & - & NDXY & PDLXY \\
\hline & Uso de herbicidas/fungicidas & - & NDRMT & NDRXY & NDRXY & PIRCT & - & NDXY & PDRXY \\
\hline \multirow{3}{*}{ Plantio } & Transplante de mudas para a lavoura & - & - & - & - & PIRCT & - & NDXY & PILCT \\
\hline & Controle de pragas & - & MDLMY & - & - & PDRCT & - & NDXY & PDLXY \\
\hline & Adubação de plantio e cobertura & - & - & - & - & PIRCT & - & NDXY & PDLXY \\
\hline \multirow{2}{*}{ Capação } & Aplicação de antibrotante & - & NDLCT & NDRXY & NDRXY & PIRCT & - & NDXY & PDLXY \\
\hline & Quebra do botão floral & - & - & - & - & PILCT & - & NDXY & PDLMT \\
\hline \multirow{2}{*}{ Colheita } & Quebra das folhas & - & - & - & - & - & - & NDXY & PILCT \\
\hline & Transporte até a propriedade & - & - & - & - & - & - & - & - \\
\hline \multirow{2}{*}{ Cura das folhas } & Preparação das varas de sustentação & - & - & - & - & PDLCT & - & NDXY & PDLCT \\
\hline & Secagem em estufa & - & NDRMT & - & - & PDRMT & - & NDXY & PDLXY \\
\hline $\begin{array}{l}\text { Classificação, } \\
\text { manocas e } \\
\text { enfartamento }\end{array}$ & $\begin{array}{l}\text { Classificação do tabaco em grupos de } \\
\text { acordo com seu preparo, sua } \\
\text { apresentação e arrumação, sua posição } \\
\text { nas plantas, cor das folhas e sua } \\
\text { qualidade. }\end{array}$ & NDLMT & & & & PDLMT & & NDXY & PDLMT \\
\hline \multirow{2}{*}{$\begin{array}{l}\text { Transporte } \\
\text { para as } \\
\text { fumageiras }\end{array}$} & $\begin{array}{l}\text { Carregamento nos veículos de } \\
\text { transporte }\end{array}$ & - & - & - & - & PILCT & - & NDXY & - \\
\hline & Transporte até as indústrias & - & - & - & - & PIRCT & - & - & - \\
\hline
\end{tabular}

LEGENDA: P - positivo; N - negativo; D - Direto; I - Indireto; L - Local; R - Regional; C - Curto; M - Médio; X - Longo; Y - Permanente; T Temporário 


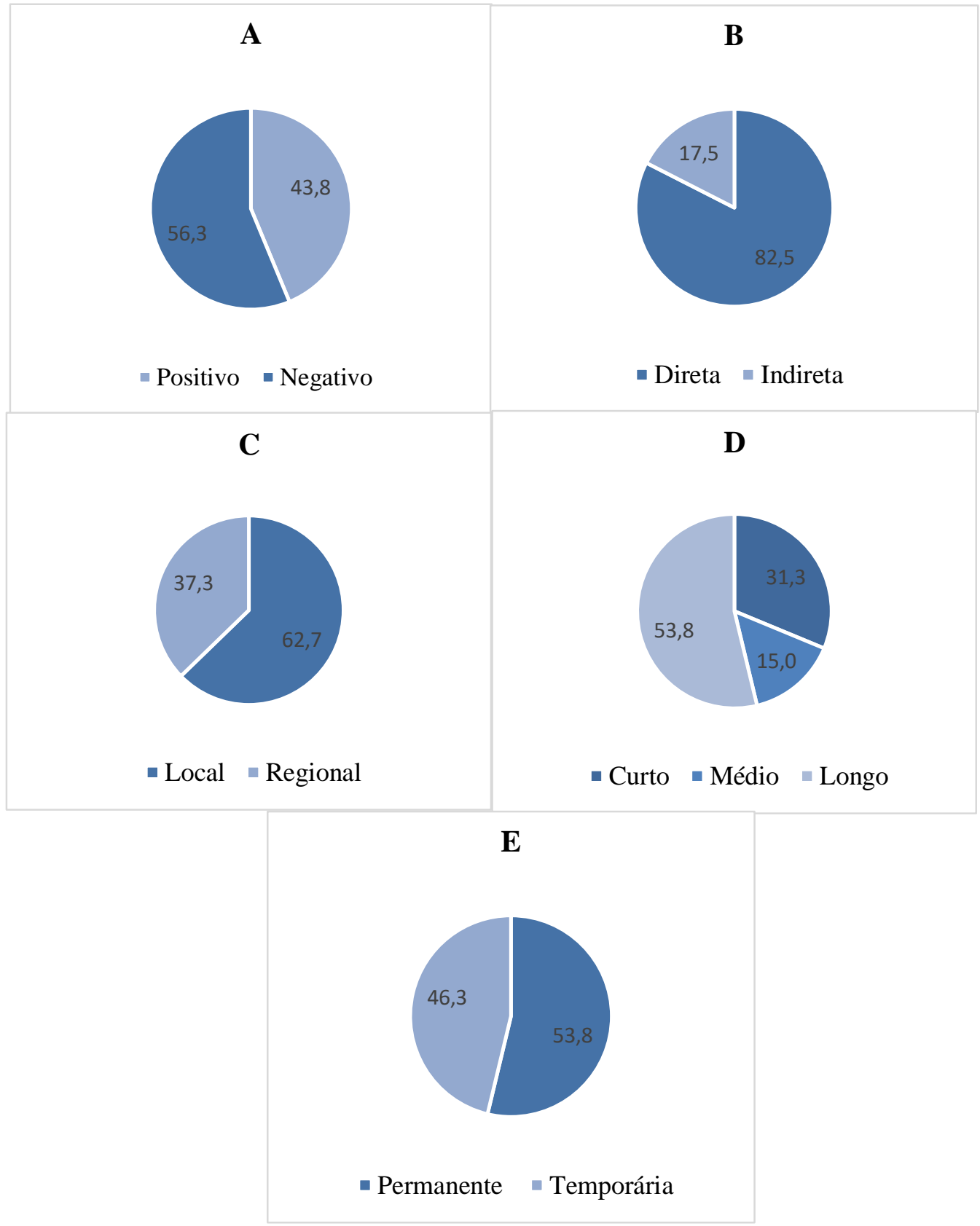

Figura 1- Gráficos gerados a partir da contagem dos efeitos elencados na Matriz de Leopold modificada Tabela 3.0 gráfico A é o de valor/impacto, B refere-se a ordem, $\mathrm{C}$ ao espaço, D ao tempo e $\mathrm{E}$ a dinâmica.

Muitas vezes, o plantio é executado em áreas impróprias. São assim consideradas as áreas próximas a cursos d'água, regiões ribeirinhas, próximas a residências ou, ainda, em zonas íngremes, facilitando a lixiviação de fertilizantes e pesticidas. Pode haver, ainda, uma ampliação da área plantada, com o desmatamento de áreas que, muitas vezes, são abrigos para ecossistemas, o que gera um impacto no meio ambiente. Estudos realizados em cursos 
d'agua adjacentes mostraram que a poluição aumenta em áreas onde há redução da área florestal adjacente [15].

Além disso, o risco de exposição do produtor à nicotina é crescente. A característica hidrossolúvel da nicotina pode ainda contaminar frutas, chás, temperos e plantas medicinais, o que pode impactar na disseminação de efeitos adversos [16,17]. Esta substância pode infiltrar-se no organismo a partir do contato com o próprio suor da pele, chegando aos órgãos internos. Do que foi estudado até agora, sabe-se que a nicotina pode causar desde infarto no miocárdio, câncer e é a principal responsável pela dependência química [4].

O contato com agrotóxicos, além de contribuir para doenças cardíacas, pulmonares, auditivas, pode também contribuir para doenças psíquicas. Diversos estudos têm relacionado a ocorrência de depressão e, consequentemente, de suicídios ao uso de agrotóxicos [18]. Muitos desses efeitos à saúde do produtor são permanentes, podendo levar a doenças que, muitas vezes, não têm cura, como o câncer. Além disso, principalmente em países com economias mais frágeis, a aplicação destes produtos se dá por meio de mochilas pulverizadoras e sem o uso de proteção, o que maximiza a absorção dos pesticidas pela pele.

A conscientização dos trabalhadores, por outro lado, deve ser reconhecida, quando se observa o número crescente destes que passaram a usar as vestimentas adequadas para o manuseio da cultura. Estudos mostram a importância de seguir as orientações técnicas de segurança do trabalho, em todas as etapas. Mesmo aqueles que somente executam a colheita, ou seja, não trabalharam na aplicação direta do produto, também ficaram expostos aos princípios ativos dos agrotóxicos. Há também estudos que associam determinadas substâncias a diagnósticos psiquiátricos em agricultores, tais como depressão e tendência suicida [19].

Além do uso dos agrotóxicos, a cultura do tabaco também demanda muitos nutrientes do solo. Quando há um grande uso de fertilizantes no solo, pode provocar, em caso de má aplicação, eutrofização de corpos hídricos pela lixiviação destes nutrientes. Estudos comprovam que a planta do tabaco demanda mais nitrogênio, fósforo e potássio do que outros tipos de plantas, ou seja, a plantação do tabaco esgota mais rapidamente a fertilidade do solo do que as demais culturas. Além disso, algumas práticas agrícolas que buscam maiores teores de nicotina e desenvolvimento foliar também agridem o solo em demasia [20].

De outro lado, deve-se considerar também o importante impacto na economia que a cultura do tabaco exerce para os pequenos e médios agricultores. Esta torna-se a alternativa viável diante da falta de recursos financeiros e de área suficiente para investir em outras culturas, como a soja ou o milho[1]. Esta atividade é a principal economia no centro do estado, e meio de sobrevivência de muitas famílias. Por isso deve ser garantido que o plantio do tabaco seja executado com bom senso e de modo sustentável e seguro [1-4].
A relação econômica - ambiental neste contexto pode ser equilibrada pelas ações conscientes dos produtores. Esta é uma cultura bem estabelecida, com inúmeros ganhos econômicos para a região, estado e país e deve ser sempre estudada para que reduza seus impactos diretos e indiretos. Alguns impactos são passíveis de controle e mitigação, desde que associada ao uso correto do solo e manuseio sustentável.

$\mathrm{Na}$ lavoura estudada ainda existem aspectos que podem ser melhorados e impactos que podem ser minimizados. Alguns são muito difíceis de serem evitados, uma vez que dependem de investimentos que ficam longe do alcance dos produtores familiares, como o uso de maquinarias para minimizar o contato direto com as folhas; cuidados para evitar a contaminação de recursos hídricos; descanso do solo, associado à necessidade de mais área disponível para plantio, prefinindo a lixiviação de nutrientes.

Além disso, os dados levantados demostram a dependência do modelo tecnológico convencional da cultura do tabaco, com consumo de agrotóxicos. Mesmo tendo interesse em diversificar a produção, a maioria dos agricultores continua vinculada ao sistema devido à garantia de compra do produto pelas empresas, o que não acontece com a maior parte dos cultivos de outras plantas que poderiam ser alternativas para a região. Neste contexto, a mudança para sistema de produção orgânica poderia ser um avanço significativo.

A produção de tabaco orgânico vem se mostrando uma alternativa interessante à produção convencional. Tal modo de produção consiste na restrição ao uso de produtos químicos, como defensivos agrícolas e fertilizantes, e também quanto à área de plantio, que deve ficar isolada de plantações convencionais para evitar contaminação. Além disso, os produtos orgânicos, como o tabaco, devem respeitar legislação vigente para serem reconhecidos como tal [21]. Experiências realizadas no Paraná mostraram que a rentabilidade da produção convencional pode ser superada com um manejo inteligente, um combinado de produção orgânica de tabaco concomitante com processamento de frutas e hortaliças [22], mostrando-se uma alternativa interessante ao modo convencional de produção de tabaco.

Portanto, com base nos dados encontrados na matriz de Leopold, foi reconhecida a existência de impacto negativo associado ao modo de produção, o que pode ser minimizado. Permitiu observar o cenário do qual os profissionais da área podem partir para propor modificações. Mostrou-se uma ferramenta adequada para reconhecer os principais impactos ambientais na cadeia do tabaco, na propriedade estudada. Com ela foi possível avaliar as interações entre os fatores e as ações e isso é de grande importância no momento em que se almeja mapeá-las. A matriz de Leopold apresentou grande flexibilidade, auxiliando de modo positivo nas avaliações dos impactos e promovendo uma maior conscientização dos problemas que devem ser monitorados a fim de minimizar impactos ambientais [23]. 


\section{Conclusões}

Através desse trabalho, foi possível verificar que os impactos existentes na propriedade produtora de tabaco avaliada foram, em sua maioria, negativos ao homem e ao meio ambiente. No entanto, existem impactos positivos que impulsionam a continuidade desta atividade agrícola e da economia regional. $\mathrm{O}$ equilíbrio tanto de impactos diretos ou indiretos, de longo ou curto prazo, negativos ou positivos, se dá pelo manuseio correto da lavoura, seguindo orientações de segurança do trabalho. Alguns impactos relacionados ao desgaste do solo e o uso intensivo de agrotóxicos e de fertilizantes são de difícil controle devido à condição de produtor agrícola familiar com pequenas propriedades. A possibilidade de mudar para um sistema orgânico torna-se um meio de redução dos impactos ambientais negativos.

\section{Agradecimentos}

O presente trabalho foi realizado com apoio da Coordenação de Aperfeiçoamento de Pessoal de Nível Superior - Brasil (CAPES) - Código de Financiamento 001. Também agradecemos aos agricultores A. Voese e N. Schlesener pela descrição do processo.

\section{CASE STUDY: EMPLOYMENT OF THE LEOPOLD MATRIX FOR THE EVALUATION OF IMPACTS ASSOCIATED WITH THE PRODUCTION OF TOBACCO ON A PROPERTY IN THE PARDO RIVER VALLEY}

ABSTRACT: The tobacco industry has been gaining prominence over the last years, however, its health consequences remain a constant concern. Considering the prominence of these industries, the management systems are essential to minimize impacts in this field. Due to the consequences that can be caused to the environment, this work aims to develop a case study of a tobacco producing property in order to determine environmental impacts using an interaction matrix. The Leopold Matrix was the model used to evaluate the biotic and anthropic effects in comparison to the environmental aspects. The results showed the most critical, important, frequent and significant points of each stage of the agricultural production process, stating that these are of extreme environmental importance considering the negative impacts on air, soil and water. In addition, workers may be exposed to the risks associated with their tobacco production activities. It is possible to highlight that the main positive aspect is related to economic activity, which is the main source of economy in the central state of Rio Grande do Sul.

Keywords: Tobacco. Environmental Impacts. Leopold Matrix.

\section{Referências}

[1] HUSSAIN, M. et al. Hazardous pollutants emissions and environmental impacts from fuelwood burned and synthetic fertilizers applied by tobacco growers in Pakistan. Environmental Technology \& Innovation, Vol. 7, p. 169-181, 2017.

[2] DE MIRANDA B. A. C. A região de Santa Cruz do Sul e o fumo: panorama de uma" especialização" nociva. Indicadores Econômicos FEE, Vol. 42, n. 1, p. 43 62,2014

[3] ETGES, V. E. et al. O impacto da cultura do tabaco no ecossistema e na saúde humana. Textual, Vol. 1, nº 1, p. 14-21, 2002.

[4] BOEIRA, S. L.; GUIVANT, J. S. Indústria de tabaco, tabagismo e meio ambiente: as redes ante os riscos. Cadernos de Ciência \& Tecnologia, Vol. 20, $\mathrm{n}^{\circ}$. 1 p. $45-78,2003$.

[5] CONTI, C. L. et al. Pesticide exposure, tobacco use, poor self-perceived health and presence of chronic disease are determinants of depressive symptoms among coffee growers from Southeast Brazil. Psychiatry research, Vol. 260, p. 187-192, 2018 .

[6] ABD EL-AZIZAA.E.M, HATERAH, A., AL-SEWAIDAN. Pesticide exposure, tobacco use, poor self-perceived health and presence of chronic disease are determinants of depressive symptoms among coffee growers from Southeast Brazil Natural radioactivity contents in tobacco. International Congress Series. Volume 1276, February 2005, Pages 407-408.

[7] DE REZENDE, L. F. M. et al. Proportion of cancer cases and deaths attributable to lifestyle risk factors in Brazil. Cancer epidemiology, Vol. 59, p. 148-157, 2019.

[8] NOVOTNY, T. E.; ZHAO, F. Consumption and production waste: another externality of tobacco use. Tobacco control, Vol. 8, n .1, p. 75-80, 1999.

[9] DE OLIVEIRA S. A. et al. Utilização de equipamentos de proteção individual e agrotóxicos por agricultores de município do recôncavo Baiano. Revista da Universidade Vale do Rio Verde, Vol. 15, n.1, p. 738-754, 2017.

[10] REICHERT, J. M., PELLEGRINI, A., RODRIGUES, M. F. Tobacco growth, yield and quality affected by soil constraints on steeplands. Industrial Crops and Products. Vol 128, p. 512-526, 2019.

[11] KAERCHER, J. A. et al. Optimization of biodiesel production for selfconsumption: considering its environmental impacts. Journal of Cleaner Production, Vol. 46, p. 74-82, 2013.

[12] PERES, F.; MOREIRA, J. C..Saúde e ambiente em sua relação com o consumo de agrotóxicos em um pólo agrícola do Estado do Rio de Janeiro, Brasil. Cadernos de Saúde Pública, Vol. 23, p. S612-S621, 2007.

[13] SENA, T. R. R.; VARGAS, M. M.; OLIVEIRA, C. C. C. Saúde auditiva e qualidade de vida em trabalhadores expostos a agrotóxicos. Ciência \& Saúde Coletiva, Vol. 18, p. 1753-1761, 2013.

[14] SOUZA, G. S. et al. Presença de agrotóxicos na atmosfera e risco à saúde humana: uma discussão para a Vigilância em Saúde Ambiental. Ciência \& Saúde Coletiva, Vol. 22, p. 3269-3280, 2017.

[15] BORTOLUZZI, E. C. et al. Contamination of surface water by pesticides as a function of soil use in the Agudo watershed, RS. Revista Brasileira De Engenharia Agricola e Ambiental, Vol. 10, n. 4, p. 881-887, 2006. 


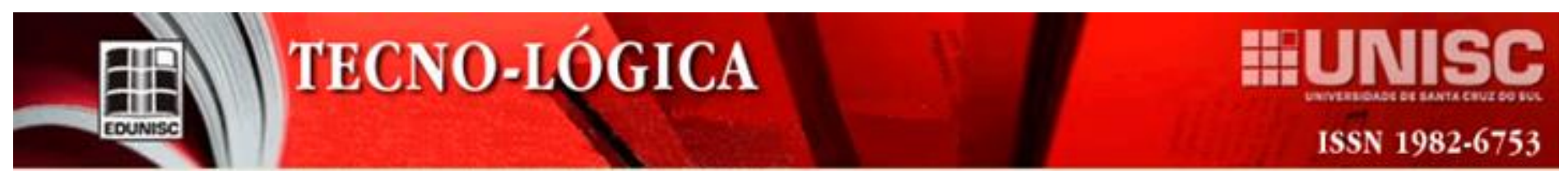

[16] SELMAR, D. et al. Uptake of nicotine from discarded cigarette butts-A so far unconsidered path of contamination of plant-derived commodities. Environmental Pollution, Vol. 238, p. 972-976, 2018.

[17] LECOURS, N. et al. Environmental health impacts of tobacco farming: a review of the literature. Tobacco control, Vol. 21, n. 2, p. 191-196, 2012.

[18] COGO, L. A. et al. Functional evaluation of the vestibular system of guinea pigs poisoned by an organophosphate through caloric stimulation. Revista CEFAC, Vol. 16, n . 5, p. 1434-1442, 2014.

[19] ARCURY, T. A.; QUANDT, S. A. Health and social impacts of tobacco production. Journal of Agromedicine, Vol 11, n. 3-4, p. 71-81, 2006.

[20] MOULA, M. S. et al. Effects of Consecutive Two Years Tobacco Cultivation on Soil Fertility status at Bheramara Upazilla in Kushtia District. J Rice Research, Vol. 6, n. 1, p. 190, 2018.

[21] MINISTÉRIO DA AGRICULTURA, PECUÁRIA E ABASTECIMENTO. Instrução normativa $n^{\circ} 17.18$ de junho de 2014.

[22] AHRENS, D.C., LLANILO, R.F., MILLÉO, R.D.S.; Possibilidades de diversificação do cultivo de fumo convencional por sistemas de produção de base agroecológica no Centro-Sul do Paraná, Brasil. Sociedade Brasileira de Economia, Administração e Sociologia Rural. Julho, 2009.

[23] POTRICH, A. L.; TEIXEIRA, C. E.; FINOTTI, A. R. Avaliação de impactos ambientais como ferramenta de gestão ambiental aplicada aos resíduos sólidos do setor de pintura de uma indústria automotiva. Estudos Tecnológicos em Engenharia, Vol. 3, nº 3, p. 162-175, 2007. 